\title{
Internal sources of water on Earth
}

\author{
Linda T. Elkins-Tanton \\ Arizona State University \\ email: lelkinst@asu.edu
}

\begin{abstract}
Rocky planets are built through a series of highly energetic accretionary impacts. The accreting bodies are thought to carry small amounts of water to the growing planet, but it is debated whether the planet can retain this water through the accretionary process, or if water needs to be added largely after the planet is complete and cooled. Most of the relatively few measurements of deuterium to hydrogen in cometary water do not match Earths water, though the single example of 103P/Hartley 2 is a good match (Mumma \& Charnley 2011, Hartogh et al. 2011). Alexander et al. (2012) point out, however, that organic materials in comets have far higher $\mathrm{D} / \mathrm{H}$ than does their water, so in bulk no comet matches Earths $\mathrm{D} / \mathrm{H}$ ratio, not even comet Hartley. Meteorites provide evidence that the material that built planets had small but sufficient amounts of water, and analysis of its isotopic composition demonstrates that water from rocky material, and not comets, provided water to the Earth (Alexander et al. 2012). This current evidence that Earths water came from rocky asteroidal material does not solve the question of whether this material was the accreting material that built the planet, or if it was added later. Mission data from the Moon and Mercury demonstrates that those bodies are not completely devoid of volatiles (McCubbin et al. 2010, Saal et al. 2008, Peplowski et al. 2011). The Moon, in particularly, has small amounts of internal water that survived its energetic origins (Hauri et al. 2011). Thus, the giant accretionary impacts that build planets do not completely dry them, and the water from their initial building blocks can be retained. If volatiles are delivered and partly retained during accretion, then the initial habitability of a young planet may be set by degassing of a magma ocean. Models predict that cooling can result in liquid water oceans within ten or tens of millions of years (Abe and Matsui 1986, Elkins-Tanton 2011). Thus, rocky planets in solar systems similarly composed to our own have a good chance of forming with water oceans, and of being habitable, at least for some period of time.
\end{abstract}

\section{Keywords.}

Rocky planets are built through a series of highly energetic accretionary impacts (Chambers 2004, Goldreich \& Ward 1973, Weidenschilling et al. 1997). At the earliest stage of gravitationally-driven impacts, planetesimals tens to hundreds of kilometers in radius collide and may accrete. The kinetic energy of these impacts is insufficient to cause significant melting. As planetary accretion continues through the oligarchic stage, planetary embryos the size of Mars collide and grow into bodies the size of Earth and beyond.

These giant accretionary impacts are energetic enough to melt their targets to some depth. The energy of these impacts, such as the Earths final giant impact that produced the Moon, raised doubts about how any water delivered by the impactor or previously existing on the target planet could be retained through the process. If water could not be retained during the oligarchic stage of accretion, then it needed to be added later. Later additions could be made either in the tail of accretion or through additions of material driven to the Earth via later solar system disturbances, for example, those posited in the Nice model (Gomes et al. 2005).

A first question is the kind of material that provided the Earths water. Was it icy, organic-rich cometary material, or rocky asteroidal material? Cometary materials was the 
leading candidate for years, until a number of comets were measured and their deuterium to hydrogen ratios were found to be far from the Earths (Mumma \& Charnley 2011). In 2011, however, Hartogh et al. (2011) demonstrated that comet 103P/Hartley 2 is a good match for Earth in deuterium to hydrogen. Alexander et al. (2012) point out, however, that organic materials in comets have far higher $\mathrm{D} / \mathrm{H}$ than does their water, so in bulk no comet matches Earths D/H ratio, not even comet Hartley.

Meteorites provide a library of material as candidates for the building blocks of the rocky planets. Almost all rocky meteorites have at least tens to hundreds of parts per million of water bound into silicate minerals, and some contain water on the percentage level (Jarosewich 1990). Analysis of the isotopic composition of water in meteorites demonstrates that chondritic planetesimals, and not comets, provided water to the Earth (Alexander et al. 2012).

The match of chondritic water to earth is consistent with water delivery and retention during the primary accretion phase of Earth, because the bulk composition of the Earth is a close match to the bulk composition of the chondrites. This consistency is necessary but not sufficient proof that Earths water was delivered during accretion. The Earth could still have been dried through the energetic processes of accretion, and then had further chondritic material added later, after primary accretion was compete.

Mission data provides evidence, however, that giant impacts do not perfectly dry their targets, and thus that the process of accretion does not end with a dry planet. Mission data from the Moon and Mercury demonstrates that those bodies are not completely devoid of volatiles (McCubbin et al. 2010, Peplowski et al. 2011, Saal et al. 2008). Mercury should be the driest of planets. Either it was formed from highly reduced material itself devoid of volatiles and with the majority of its iron in metallic form (Weidenschilling 1978), or, in the more currently widely accepted hypothesis, it suffered an immense glancing impact that stripped off much of its silicate mantle and left it with an unusually large core for its total radius (Asphaug \& Reufer 2014). Under either scenario Mercury should have been left devoid of volatile elements, according to some researchers, but the MESSENGER mission demonstrated that it still contains significant volatile elements (Peplowski et al. 2011).

The Moon was long thought to be dry to the parts per million level. In 2008, small amounts of water were measured in beads of volcanic glass that erupted from the lunar interior (Saal et al. 2008), and further measurements of other volcanic material demonstrated that parts of the interior of the Moon have water in similar quantities to parts of the Earths mantle (Hauri et al. 2011). Though the bulk Moon likely started with at least an order of magnitude less water than the bulk Earth (Elkins-Tanton \& Grove 2011), its bulk material was not dried by the giant impact and subsequent processing in a disk around the Earth. In fact, completely drying materials is difficult. Hydroxyls bond readily to many materials and even small gas pressures in a disk will retain some level of water. Thus, the giant accretionary impacts that build planets do not completely dry them, and the water from their initial building blocks can be retained.

If volatiles are delivered and partly retained during accretion, then degassing of a magma ocean may set the initial habitability of a young planet. Models predict that cooling can result in liquid water oceans within ten or tens of millions of years (Abe \& Matsui 1985, Elkins-Tanton 2008, 2011, Zahnle et al. 1988). In fact, growing rocky planets likely experienced serial magma oceans due to the number of giant impacts required to build the planet. Solidification of a magma ocean on an Earth-sized planet under a thin atmosphere takes not more than a few millions years (Elkins-Tanton 2008). Thus most giant impacts would strike a solid planet with a steam atmosphere. The majority of an 
atmosphere is retained even during many giant impacts, however (Genda \& Abe 2003, 2005).

Evidence from isotopes shows that Earths water came from rocky chondritic materials. Evidence from missions to Mercury and to the Moon indicates that giant impacts do not perfectly dry their targets. Modeling indicates that giant impacts to not commonly strip the entire existing atmosphere off a growing planet. Thus, rocky planets are likely to be born with enough water for at least thin oceans and thick atmospheres.

Later additions of water from both comets and rocky asteroidal material are inevitable and indeed are ongoing to the present. It is not, however, necessary for the production of a planet with a quantity of water sufficient for habitability simply through primary accretion. This finding indicates that sufficient water is a likely result of accretion of all rocky planets. Later stochastic processes are not necessary to deliver water. Thus, rocky planets in solar systems similarly composed to our own have a good chance of forming with water oceans, and of being habitable, at least for some period of time.

\section{References}

Abe, Y. \& Matsui, T. 1985. The formation of an impact-generated $\mathrm{H}_{2} \mathrm{O}$ atmosphere and its implications for the thermal history of the Earth. Journal of Geophysical Research 90, 545-59

Abe, Y. \& Matsui, T. 1986. Early evolution of the Earth: accretion, atmosphere formation, and thermal history. Journal of Geophysical Research 91, E291-E302

Alexander, C.M.O'D., Bowden, R., Fogel, M. L., Howard, K. T., Herd, C.D.K., \& Nittler, L.R. 2012. The Provenances of Asteroids, and Their Contributions to the Volatile Inventories of the Terrestrial Planets. Science 337, 721-3

Asphaug, E. \& Reufer, A. 2014. Mercury and other iron-rich planetary bodies as relics of inefficient accretion. Nature Geoscience 7, 564-8

Chambers, J. E. 2004. Planetary accretion in the inner Solar System. Earth and Planetary Science Letters 223, 241-52

Elkins-Tanton, L T 2008. Linked magma ocean solidification and atmospheric growth for Earth and Mars. Earth and Planetary Science Letters 271, 181-91

Elkins-Tanton, L T 2011. Formation of early water oceans on rocky planets. Astrophysics and Space Science 302, 359

Elkins-Tanton, L. T. \& Grove, T L 2011. Water (hydrogen) in the lunar mantle: Results from petrology and magma ocean modeling. Earth and Planetary Science Letters 307, 173-9

Genda, H. \& Abe, Y. 2003. Survival of a proto-atmosphere through the stage of giant impacts: the mechanical aspects. Icarus 164: 149-62

Genda, H. \& Abe, Y. 2005. Enhanced atmospheric loss on protoplanets at the giant impact phase in the presence of oceans. Nature $433,842-4$

Goldreich, P., Ward, W R 1973. The formation of planetesimals. ApJ 183, 1051-61

Gomes, R., Levison, H. F., \& Tsiganis, K., Morbidelli, A. 2005. Origin of the cataclysmic Late Heavey Bombardment period of the terrestrial planets. Nature 435, 466-9

Hartogh, P., Lis, D. C., Bockelee-Morvan, D., de Val-Borro, M., Biver, N., Kuppers, M., Emprechtinger, M., Bergin, Ed. A., Crovisier, J., Rengel, M., Moreno, R., Szutowicz, S., \& Blake, G A 2011. Ocean-like water in the Jupiter-family comet 103P/Hartley 2. Nature $478,218-20$

Hauri, E. H., Weinreich, T., Saal, A. E., Rutherford, M. C., \& Van Orman, J. A. 2011. High Pre-Eruptive Water Contents Preserved in Lunar Melt Inclusions. Science 333, 213-5

Jarosewich, E. 1990. Chemical analyses of meteorites: A compilation of stony and iron meteorite analyses. Meteoritics 25, 323-37

McCubbin, F. M., Steele, A., Hauri, E. H., Nekvasil, H., Yamashita, S., \& Hemley, R. J. 2010. Nominally hydrous magmatism on the Moon. Proceedings of the National Academy of Sciences 107, 11223-8 
Mumma, M. J. \& Charnley, S. B. 2011. The chemical composition of comets-emerging taxonomies and natal heritage. ARAA 49, 471-524

Peplowski, P. N., Evans, L. G., Hauck, S. A., McCoy, T. J., Boynton, W. V., Gillis-Davis, J. J., Ebel, D. S., Goldsten, J. O., Hamara, D. K., Lawrence, D. J., McNutt, R. L., Nittler, L. R., Solomon, S. C., Rhodes, E. A., Sprague, A. L., Starr, R. D. \& Stockstill-Cahill, K. R. 2011. Radioactive Elements on Mercury's Surface from MESSENGER: Implications for the Planet's Formation and Evolution. Science 333, 1850-2

Saal, A. E., Hauri, E. H., Lo Cascio, M., Van Orman, J. A., Rutherford, M. J., \& Cooper, R. F. 2008. Volatile content of lunar volcanic glasses and the presence of water in the Moon's interior. Nature 454, 193-5

Weidenschilling, S. J. 1978. Iron/Silicate Fractionation and the Origin of Mercury. Icarus 35, 99-111

Weidenschilling, S. J., Spaute, D., Davis, D. R., Marzari, F., \& Ohtsuki, K. 1997. Accretional evolution of a planetesimal swarm. Icarus 128, 429-55

Zahnle, K. J., Kasting, J. F., \& Pollack, J. B. 1988. Evolution of a steam atmosphere during Earth's accretion. Icarus 74, 62-97 